DOI: $10.17516 / 1997-1397-2020-13-4-439-450$

УДК 536.423 .1

\title{
To the Question of Analytical Estimate of Evaporation Time of the Drop, Crossing Through the Heat Media
}

\author{
Sergey O. Gladkov* \\ Moscow Aviation Institute \\ Moscow, Russian Federation
}

Received 01.02.2020, received in revised form 13.03.2020, accepted 20.05.2020

\begin{abstract}
Due to the kinetic approach the modelling description of the drop evaporation is offer. The main equation of the theory received due to the conservation law of dissipative functions of the vapor-liquid system. The diapason of drop size it's finding when its stability. It's comparison of the results with the famous classical is given. The numerical estimate of the linear size of small disperse phase when take place usually evaporation (i.e. the Knudsen's number is a small $K n=\frac{l}{R} \ll 1$, where $l$ is a free length path of the molecule and $R$ is an drop radius) are given.
\end{abstract}

Keywords: dissipative function, evaporation, free length path.

Citation: S.O. Gladkov, To the Question of Analytical Estimate of Evaporation Time of the Drop, Crossing Through the Heat Media, J. Sib. Fed. Univ. Math. Phys., 2020, 13(4), 439-450.

DOI: 10.17516/1997-1397-2020-13-4-439-450.

The task that will be discussed in this article is not new, and has about a century of backstory. It must be said that for many physical tasks devoted to the study of the properties of fine environments (fogs, steam, smoke, dust, etc.), it is characteristic that their solution in the vast majority of cases has an empirical and experimental character. Although the number of theoretical works in this direction has been growing quite rapidly in recent years, the conclusion of the main equations is usually based on the dependents obtained purely experimentally. In this paper, we will move away from the well-established stereotype of problem-solving in this direction, and use the general principles of the theory of non-equilibrium processes, using as the basic method of describing the dissipative function $\dot{Q}=T \dot{S}$, where $T$ is an equilibrium temperature, $S$ is an entropy, and the "point" under the letter as usually shows the differentiation in the time.

\section{The conclusion of the main equation}

Let's write the balance equation taking into account the interaction of gas phase molecules and molecules in a drop at the edge of their contact in the form of the next amount of dissipative functions

$$
T \frac{d}{d t} \int_{V_{1}} s_{1} d V+T \frac{d}{d t} \int_{V-V_{1}} s_{2} d V+\frac{d}{d t} \int_{\sigma} \alpha d \sigma=0,
$$

where $s_{1}$ is an entropy of drop in the unite of its volume, $s_{2}$ is an entropy is a unit of volume surrounding the gas phase drop, including molecules of the already evaporated drop matter, $V_{1}$

*sglad51@mail.ru

(c) Siberian Federal University. All rights reserved 
variable drop volume, $V=V_{1}+V_{2}=$ const fullvolume occupied by drop and gas, $\sigma$ the surface area of the drop. By performing a simple time-by-time differentiation, we find

$$
T \dot{S}_{1}+T s_{1} \dot{V}_{1}+T \dot{S}_{2}-T s_{2} \dot{V}_{1}+\alpha \dot{\sigma}_{1}=0 .
$$

Introducing here the hidden warmth of steam formation

$$
\Delta Q_{V}=T\left(s_{1}-s_{2}\right) .
$$

Getting out (2)

$$
T \dot{S}_{1}+T \dot{S}_{2}+\Delta Q_{V} \dot{V}_{1}+\alpha \dot{\sigma}_{1}=0
$$

Our task now is to calculate the first two components that are part of the equation (4). According to the definition of entropy in the language of the distribution function (see [1]), we have

$$
S_{1}=-\frac{1}{Z_{1}} \int n_{1} \ln n_{1} d^{3} p,
$$

where $n_{1}$ is an nonequilibrium function of the distribution of fluid molecules by pulses, and the rationing multiplier

$$
Z_{1}=\int \bar{n}_{1} d^{3} p
$$

where the equilibrium distribution function

$$
\bar{n}_{1}=\exp \left(-\frac{\varepsilon_{1}(p)-\mu_{1}(P, T)}{T}\right) .
$$

Note here that Boltzmann's constant in (5) and in (7) and beyond we will believe an equal unit. Kinetic energy of molecules in liquid is $\varepsilon_{1}(p)=\frac{p^{2}}{2 m_{1}}$, where $\mu_{1}(P, T)$ is the chemical potential of a liquid molecules in drop. Similarly

$$
S_{2}=-\frac{1}{Z_{2}} \int n_{2} \ln n_{2} d^{3} p
$$

where $Z_{2}=\int \bar{n}_{2} d^{3} p$,

$$
\bar{n}_{2}=\exp \left(-\frac{\varepsilon_{2}(p)-\mu_{2}(P, T)}{T}\right),
$$

where $\varepsilon_{2}(p)=\frac{p^{2}}{2 m_{2}}$ is the kinetic energy of gas molecules, and $\mu_{2}(P, T)$ their chemical potential. Differentiating (5) and (8) on time, we have, lowering the permanent term

$$
\dot{S}_{1}=-\frac{1}{Z_{1}} \int \dot{n}_{1} \ln n_{1} d^{3} p, \quad \dot{S}_{2}=-\frac{1}{Z_{2}} \int \dot{n}_{2} \ln n_{2} d^{3} p .
$$

In the accordance with Boltzmann's kinetic equation, we have the right to write down that

$$
\dot{n}_{1}=L_{1}\left(n_{1}, n_{2}\right), \quad \dot{n}_{2}=L_{2}\left(n_{2}, n_{1}\right),
$$

where $L_{1}\left(n_{1}, n_{2}\right)$ and $L_{2}\left(n_{2}, n_{1}\right)$ respectively, the integrals of the collisions of liquid and gas molecules at the border of their contact. Therefore, with the account of expressions (10) and (11) the equation (4) will take the form

$$
-\frac{T}{Z_{1}} \int L_{1}\left(n_{1}, n_{2}\right) \ln n_{1} d^{3} p-\frac{T}{Z_{2}} \int L_{2}\left(n_{2}, n_{1}\right) \ln n_{2} d^{3} p+\Delta Q_{V} \dot{V}_{1}+\alpha \dot{\sigma}_{1}=0 .
$$


The solution of kinetic equations we will look for in the so-called "tau- approximation", according to which the integrals of collisions are replaced by the approximation of expression

$$
L_{1} \approx-\frac{\delta n_{1}}{\tau_{12}}, \quad L_{2} \approx-\frac{\delta n_{2}}{\tau_{21}},
$$

where $\tau_{12}$ the relaxation time of the liquid molecules when they are scattered on gas molecules, and $\tau_{21}$ the relaxation time of the gas molecules when they are scattered on liquid molecules. It is clear that these times are different. We will now find amendments $\delta n_{1,2}$ to the distribution function due to the interaction. According to the kinetic equation, we have

$$
\dot{n}_{1}=\frac{\partial n_{1}}{\partial t}+\mathbf{v} \cdot \nabla n_{1}+\mathbf{F} \cdot \frac{\partial n_{1}}{\partial \mathbf{p}}=-\frac{n_{1}-\bar{n}_{1}}{\tau_{12}} .
$$

As we are looking for a stationary solution $\frac{\partial n_{1}}{\partial t}=\frac{\partial n_{2}}{\partial t}=0$. To the rum of that, it should be considered that strength $\mathbf{F}=0$. As a result

$$
\mathbf{v} \cdot \nabla n_{1}=-\frac{n_{1}-\bar{n}_{1}}{\tau_{12}}
$$

And similarly

$$
\mathbf{v} \cdot \nabla n_{2}=-\frac{n_{2}-\bar{n}_{2}}{\tau_{21}}
$$

We will look for solutions to equations (15) and (16) by the method of successive approximations, that is, let's put that

$$
n_{1}=\bar{n}_{1}+\delta n_{1}, \quad n_{2}=\bar{n}_{2}+\delta n_{2} .
$$

That's why we get

$$
\begin{aligned}
& \mathbf{l}_{12} \cdot \delta n_{1}+\delta n_{1}=-\mathbf{l}_{12} \cdot \nabla \bar{n}_{1}, \\
& \mathbf{l}_{21} \cdot \delta n_{2}+\delta n_{2}=-\mathbf{l}_{21} \cdot \nabla \bar{n}_{2} .
\end{aligned}
$$

where free-range vectors are introduced $\mathbf{l}_{12}=\mathbf{v} \tau_{12}, \mathbf{l}_{21}=\mathbf{v} \tau_{21}$. The solution of equations (18) is convenient to look for by decomposition of the desired functions in the integral Fourier. Indeed, have for arbitrary (yet) function

$$
f(\mathbf{r})=\int_{-\infty}^{\infty} \exp (i \mathbf{k r}) f_{\mathbf{k}} \frac{d^{3} \mathbf{k}}{(2 \pi)^{3}}
$$

where by one-dimensional integral we mean three-dimensional integral, $f_{\mathbf{k}}$ is the Fourier image of the function $f$. Substituting (19) in any of the equations (18), easy find

$$
\int(1+i \mathbf{k} \mathbf{l}) \delta n_{\mathbf{k}} \frac{d^{3} \mathbf{k}}{(2 \pi)^{3}}=-\mathbf{l} \cdot \nabla \int \bar{n}_{\mathbf{k}} \exp (i \mathbf{k r}) \frac{d^{3} \mathbf{k}}{(2 \pi)^{3}}
$$

From where

$$
\delta n_{\mathbf{k}}=-i \frac{(\mathbf{k} \cdot \mathbf{l}) \bar{n}_{\mathbf{k}}}{1+i \mathbf{k} \cdot \mathbf{l}}
$$

where $\bar{n}_{\mathbf{k}}$ is Fourier image of the equilibrium function of molecule distribution $\bar{n}(\mathbf{r})$. Substituting now the solution (20) in the definition (19), find the amendment of interest to the equilibrium function of distribution

$$
\delta n=-\frac{i}{(2 \pi)^{3}} \int \frac{(\mathbf{k} \cdot \mathbf{l}) \bar{n}_{\mathbf{k}}}{1+i \mathbf{k} \cdot \mathbf{l}} \exp (i \mathbf{k r}) d^{3} \mathbf{k}
$$


Here and beyond we simplify the recording of the Integral Fourier, lowering the limits of integration. To calculate the resulting integral, it is convenient to use the next artificial technique. Let's imagine the function $\frac{1}{1+i \mathbf{k} \cdot \mathbf{l}}$ in the form of an integral

$$
\frac{1}{1+i \mathbf{k} \cdot \mathbf{l}}=\int_{0}^{\infty} \exp (-x(1+i \mathbf{k} \cdot \mathbf{l})) d x
$$

Then from (21) it follows

$$
\delta n=-\frac{i}{(2 \pi)^{3}} \int_{0}^{\infty} \exp (-x) d x \int(\mathbf{k} \cdot \mathbf{l}) \bar{n}_{\mathbf{k}} \exp (i \mathbf{k}(\mathbf{r}-x \mathbf{l})) d^{3} \mathbf{k}
$$

Next, as

$$
\bar{n}_{\mathbf{k}}=\int \bar{n}\left(\mathbf{r}^{\prime}\right) \exp \left(-i \mathbf{k} \mathbf{r}^{\prime}\right) d^{3} \mathbf{r}^{\prime},
$$

then substituting (24) in the solution (23), will have as a result of a simple regrouping of multipliers

$$
\delta n=-\frac{i}{(2 \pi)^{3}} \int_{0}^{\infty} \exp (-x) d x \int \bar{n}\left(\mathbf{r}^{\prime}\right) d^{3} \mathbf{r}^{\prime} \int(\mathbf{k} \cdot \mathbf{l}) \exp \left(i \mathbf{k}\left(\mathbf{r}-\mathbf{r}^{\prime}-x \mathbf{l}\right)\right) d^{3} \mathbf{k} .
$$

To calculate the internal integral, let's use the following technique. Let's write it down as

$$
\int \exp (i \mathbf{k}(\mathbf{R}-\mathbf{l} x))(\mathbf{k} \mathbf{l}) d^{3} \mathbf{k}=i \frac{\partial}{\partial x} \int \exp (i \mathbf{k}(\mathbf{R}-\mathbf{l} x)) d^{3} \mathbf{k}=i(2 \pi)^{3} \frac{\partial}{\partial x} \delta(\mathbf{R}-\mathbf{l} x),
$$

where radius-vector $\mathbf{R}=\mathbf{r}-\mathbf{r}^{\prime}$. As a result, from (25) it follows

$$
\delta n=\int_{0}^{\infty} \exp (-x) d x \frac{\partial}{\partial x} \int \bar{n}\left(\mathbf{r}^{\prime}\right) \delta\left(\mathbf{r}-\mathbf{r}^{\prime}-\mathbf{l} x\right) d^{3} \mathbf{r}^{\prime}=\int_{0}^{\infty} \exp (-x) d x \frac{\partial}{\partial x} \bar{n}(\mathbf{r}-\mathbf{l} x) d x
$$

We will take the resulting integral by means of integration piece by piece. In fact,

$$
\delta n=\int_{0}^{\infty} \exp (-x) d x \frac{\partial}{\partial x} \bar{n}(\mathbf{r}-\mathbf{l} x) d x=\int_{0}^{\infty} \exp (-x) \bar{n}(\mathbf{r}-\mathbf{l} x) d x-\bar{n}(\mathbf{r}) .
$$

Remembering now the operator of the broadcast, namely the rule

$$
\bar{n}(\mathbf{r}-\mathbf{l} x)=\exp (-x \mathbf{l} \cdot \nabla) \bar{n}(\mathbf{r})
$$

Find out (26)

$$
\delta n=\int_{0}^{\infty} \exp (-x(1+\mathbf{l} \cdot \nabla)) \bar{n}(\mathbf{r})-\bar{n}(\mathbf{r}) .
$$

Therefore, for the amendments we are in, we get such solutions to equations (18)

$$
\left\{\begin{array}{l}
\delta n_{1}=\int_{0}^{\infty} \exp \left(-x\left(1+\mathbf{l}_{12} \cdot \nabla\right)\right) \bar{n}_{1}(\mathbf{r})-\bar{n}_{1}(\mathbf{r}), \\
\delta n_{2}=\int_{0}^{\infty} \exp \left(-x\left(1+\mathbf{l}_{21} \cdot \nabla\right)\right) \bar{n}_{2}(\mathbf{r})-\bar{n}_{2}(\mathbf{r}) .
\end{array}\right.
$$

And hence, according to (12) and (13) find

$$
\frac{T}{Z_{1}} \int \frac{\delta n_{1}}{\tau_{12}} \ln \bar{n}_{1} d^{3} p+\frac{T}{Z_{2}} \int \frac{\delta n_{2}}{\tau_{21}} \ln \bar{n}_{2} d^{3}+\Delta Q_{V} \dot{V}_{1}+\alpha \dot{\sigma}_{1}=0
$$


where the amendments $\delta n_{1}, \delta n_{2}$ accurate solutions (28), albeit in tau approximation. By determining the equilibrium functions of distribution (7) and (9) of (29) the dissipative balance equation follows

$$
-\frac{T}{Z_{1}} \int \frac{\varepsilon_{1}-\mu_{1}}{\tau_{12}} \delta n_{1} d^{3} p-\frac{T}{Z_{2}} \int \frac{\varepsilon_{2}-\mu_{2}}{\tau_{21}} \delta n_{2} d^{3} p+\Delta Q_{V} \dot{V}_{1}+\alpha \dot{\sigma}_{1}=0 .
$$

Note that the last (30) is also convenient to present as $\int \alpha d S=\bar{\varepsilon}_{1} N_{1}=\bar{\varepsilon}_{1} \int c_{1} d V_{1}$, where $\bar{\varepsilon}_{1}$ some medium energy coming from one particle of liquid, $c_{1}$ is their concentration. In the accordance with (28) the solution can be written down in the form of an endless series of

$$
\begin{aligned}
\delta n=\int_{0}^{\infty} \exp (-x & (1+\mathbf{l} \cdot \nabla)) \bar{n}(\mathbf{r}) d x-\bar{n}= \\
& =\int_{0}^{\infty} \exp (-x)\left(1-x \mathbf{l} \cdot \nabla+\frac{x^{2}}{2}(\mathbf{l} \cdot \nabla)^{2}-\frac{x^{3}}{3 !}(\mathbf{l} \cdot \nabla)^{3}+\ldots\right) \bar{n}(\mathbf{r}) d x-\bar{n} .
\end{aligned}
$$

Integrating here each of the material on, we come to the next decision (see [3])

$$
\begin{aligned}
\delta n & =\left[1-\mathbf{l} \cdot \nabla+(\mathbf{l} \cdot \nabla)^{2}-(\mathbf{l} \cdot \nabla)^{3}+\ldots\right] \bar{n}-\bar{n}= \\
& =\left[-\mathbf{l} \cdot \nabla+(\mathbf{l} \cdot \nabla)^{2}-(\mathbf{l} \cdot \nabla)^{3}+(\mathbf{l} \cdot \nabla)^{4} \ldots\right] \bar{n},
\end{aligned}
$$

where the shortness of the decision record (28) is presented with a single designation $\delta n$ and l, i.e. $\delta n=\left\{\delta n_{1}, \delta n_{2}\right\}$ and $\mathbf{l}=\left\{\mathbf{l}_{12}, \mathbf{l}_{21}\right\}$. If you now put the solution (31) in the balance equation (30), $(\mathbf{l} \cdot \nabla)$ then thanks to the integration of momentum all odd degrees will disappear, and instead (30) we get

$$
\begin{aligned}
& -\frac{T}{Z_{1}} \int \frac{\varepsilon_{1}-\mu_{1}}{\tau_{12}}\left[\left(\mathbf{l}_{12} \cdot \nabla\right)^{2}+\left(\mathbf{l}_{12} \cdot \nabla\right)^{4}+\left(\mathbf{l}_{12} \cdot \nabla\right)^{6} \ldots\right] \bar{n} d^{3} p- \\
& -\frac{T}{Z_{2}} \int \frac{\varepsilon_{2}-\mu_{2}}{\tau_{21}}\left[\left(\mathbf{l}_{21} \cdot \nabla\right)^{2}+\left(\mathbf{l}_{21} \cdot \nabla\right)^{4}+\left(\mathbf{l}_{21} \cdot \nabla\right)^{6} \ldots\right] \bar{n} d^{3} p+\Delta Q_{V} \dot{V}_{1}+\alpha \dot{\sigma}_{1}=0 .
\end{aligned}
$$

Leaving in (32) only square length of free run components, and given the clear kind of equilibrium function of molecule distribution (7), (9), as a result of elementary differentiation come to such an equation

$$
\begin{aligned}
& -\frac{T-\varepsilon_{1}-\mu_{1}}{T} \frac{l_{12}^{2}}{\tau_{12}}\left(\Delta \mu_{1}+\frac{\left(\nabla \mu_{1}\right)^{2}}{T}\right)- \\
& -\frac{T-\mu_{2}}{T} \frac{l_{21}^{2}}{\tau_{21}}\left(\Delta \mu_{2}+\frac{\left(\nabla \mu_{2}\right)^{2}}{T}\right)+\Delta Q_{V} \dot{V}_{1}+\left.\alpha \dot{S}\right|_{r=R}=0 .
\end{aligned}
$$

Since at the border of the two phases in the absence of chemical reactions must be met the condition of continuity of entropy, it is quite clear that there is equality

$$
\Delta Q_{V}=\left.T\left(s_{1}-s_{2}\right)\right|_{r=R}=0 .
$$

As we can see, this condition is true if the temperature is constant. However, it is quite clear that the equality of entropy at the border of the contact of the drop and gas mixture does not mean the equality of their specific heat-intensiveness, because from the point of view of mathematics equality (34) should be recorded in a slightly different form, namely how

$$
\left.s_{1}\right|_{r=R-0}=\left.s_{2}\right|_{r=R+0} .
$$


That is, the limits are taken to the left and right of the contact boundary. Therefore, it is quite clear that due to the lumpy smoothness of entropy (35) follows and condition for temperature derivatives from entropy, which just characterizes the heat intensity of both phases. Formally, this means that there is equality

$$
\left.c_{1}\right|_{r=R-0}=\left.c_{2}\right|_{r=R+0}+\Delta c,
$$

where the heat capacity supplement $\Delta c$ means the final spike in heat capacity at the edge of the section of the two environments, and the isobaric heat intensity is introduced here in accordance with the generally accepted definition [2] $c_{i}=T\left(\frac{\partial s_{i}}{\partial T}\right)_{P}$, where index is $i=1,2$.

As for the physical side of the equation (33), it should be stressed immediately that as soon as we introduce the concept of variable entropy, we automatically move on to taking into account the dissipative properties of the matter. That is, in an nonequilibrium case, which is described by the equation (33), has a condition of increasing entropy ( $H$ - Boltzmann's famous theorem). As it becomes clear now, taking into account the interaction between molecules of both phases, that is, the transfer of energy from water molecules to gas molecules and vice versa leads to the destruction of the weak surface tension of the drop. To analytically describe this process, it is necessary to focus on the remarkable property of any natural physical phenomenon, like the hierarchy of relaxation times [4].

Indeed, by the order of magnitude, the free path of molecules in the liquid $l_{12}$ is much less than the free path of gas molecules $l_{21}$, that is, inequality is performed $l_{12} \ll l_{21}$.

This means that in terms of the hierarchy of times by virtue of the condition $\tau_{12} \ll \tau_{21}$, which actually follows from a condition $\bar{n}_{1} \gg \bar{n}_{2}$, where $\bar{n}_{1}, \bar{n}_{2}$ accordingly the average concentrations of liquid and gas molecules, the basic evaporation process belongs to the first composed (33), and it is this important fact that allows us to neglect the second term.

Otherwise. The first process, as the fastest, has already occurred and the drop has begun to evaporate, and the second has not yet had time to begin. This does not mean, however, that it does not contribute to the evaporation process: in a later period of time, this contribution will appear. So, given the continuity of entropy at the contact boundary (35) and with all that said, we get this equation from (33)

$$
-\frac{T-\bar{\varepsilon}_{1}-\mu_{1}}{T} \frac{l_{12}^{2}}{\tau_{12}}\left(\Delta \mu_{1}+\frac{\left(\nabla \mu_{1}\right)^{2}}{T}\right)+\left.\alpha \dot{S}\right|_{r=R}=0 .
$$

Note also that for the chemical potentials of both phases at the border there is a condition of equilibrium

$$
\left.\mu_{1}\right|_{r=R}=\left.\mu_{2}\right|_{r=R} .
$$

Because $\dot{S}=8 \pi \alpha R \dot{R}$, of (37) find

$$
8 \pi \alpha R \dot{R}=\frac{\mu_{1}-\bar{\varepsilon}_{1}-T}{T} \frac{l_{12}^{2}}{\tau_{12}}\left(\Delta \mu_{1}+\frac{1}{T}\left(\frac{\partial \mu_{1}}{\partial R}\right)^{2}\right) .
$$

Because the distribution of heterogeneous chemical potential in contact between the two media (see [5]) describing due to the equation

$$
\Delta \mu+\frac{\mu}{\delta^{2}}-\frac{\xi \mu^{3}}{\delta^{2} T^{2}}=0,
$$


where $\delta$ is the length of heterogeneity that satisfies inequality $\delta \ll l_{\min }$, where $l_{\min }=$ $=\min \left\{l_{12}, l_{21}\right\}$, and $\xi$ is some coefficient leading to a correct decision (41), then in onedimensional case out of (40) we will get

$$
\mu(r)=\frac{\mu_{1}+\mu_{2}}{2}-\frac{\mu_{1}-\mu_{2}}{2} \operatorname{th}\left(\frac{\delta r}{\delta}\right) .
$$

Therefore, at the border of contact we have

$$
\left.\frac{\partial \mu}{\partial r}\right|_{r=R}=\frac{\mu_{2}-\mu_{1}}{2 \delta}
$$

Thus, the equation (42) takes on the form of

$$
8 \pi \alpha R \dot{R}=-\frac{\mu_{1}+\bar{\varepsilon}_{1}-T}{T} \frac{l_{12}^{2} \mu_{1}}{\tau_{12} \delta^{2}}\left(\frac{\mu_{1}}{4 T}\left(1-\frac{\mu_{2}}{\mu_{1}}\right)^{2}+\frac{\xi \mu_{1}^{2}}{T^{2}}-1\right) .
$$

Where do we get a direct integration, taking into account the initial conditions $R(0)=R_{0}$

$$
R=\sqrt{R_{0}^{2}-D_{T} t}
$$

where the diffusion coefficient is

$$
D_{T}=\frac{\mu_{1}+\bar{\varepsilon}_{1}-T}{4 \pi \alpha T} \frac{l_{12}^{2} \mu_{1}}{\tau_{12} \delta^{2}}\left(\frac{\mu_{1}}{4 T}\left(1-\frac{\mu_{2}}{\mu_{1}}\right)^{2}+\frac{\xi \mu_{1}^{2}}{T^{2}}-1\right) .
$$

And hence the time of evaporation of the liquid drop is from here from the condition of equality of zero subdivided expression, that is,

$$
t_{\text {evap }}=\frac{R_{0}^{2}}{D_{T}}
$$

As to the time of relaxation $\tau_{12}$ it is easy to show that it can be calculated by formula

$$
\frac{1}{\tau_{12}}=\frac{2 r_{2}^{2} \bar{n}_{1}}{3 \pi \sqrt{2 \pi}} \frac{m_{1}^{2} \bar{\mu}_{1}}{\left(m_{1}+m_{2}\right)^{3}} \sqrt{\frac{m_{2}}{T}},
$$

where $r_{2}$ is a radius of a molecule of the gas, $\bar{\mu}_{1}$ their medium chemical potential, $m_{1}$ is the mass of molecule of the water, $m_{2}$ is the mass of the molecule of the gas, $\bar{n}_{1}$ is the middle concentration of molecules of the water. In order of magnitude $(47)$ it follows that $\tau_{12} \approx 10^{-10} \mathrm{~s}$. A similar formula has a place for relaxation time $\tau_{21}$. It comes from a formula (47) formally replacing indices " $1^{\prime \prime}$ with " $2^{\prime \prime}$. In the order of magnitude $\tau_{21} \approx 10^{-8} \mathrm{~s}$. Calculating the evaporation time of the formula (45) also requires substitution of the chemical potential of gas and liquid. Based on the general definition of the average energy of a large particle statistical system, namely $\Omega=\mu(P, T) N$, where $N$ is a number of particles in the system, for its differential we have

$$
d \Omega=\left(\frac{\partial \mu}{\partial T}\right)_{P} N d T+\left(\frac{\partial \mu}{\partial P}\right)_{T} N d P+\mu d N
$$

According to [2], for example, in the variable $(T, P, N)$ the Helmholtz's energy differential is

$$
d \Phi=-S d T+V d P+\mu d N
$$


From the comparison (48) and (49) we see that

$$
S=-N\left(\frac{\partial \mu}{\partial T}\right)_{P}, \quad V=N\left(\frac{\partial \mu}{\partial P}\right)_{T} .
$$

It is known from [2] the one that entropy per particle can be calculated as

$$
s=\frac{S}{N}=-\frac{1}{Z} \int n \ln n d^{3} \mathbf{p}
$$

where the rationing multiplier $Z=\int \bar{n} d^{3} \mathbf{p}$, and

$$
\bar{n}=\exp \left(-\frac{\varepsilon(p)-\mu}{T}\right)
$$

is the equilibrium Maxwell distribution function, p momentum of molecule. Neglecting in (51) molecule scattering processes, we have

$$
S=-\frac{N}{Z} \int \bar{n} \ln \bar{n} d^{3} \mathbf{p}=\frac{N}{Z T} \int(\varepsilon-\mu) \exp \left(-\frac{\varepsilon-\mu}{T}\right) d^{3} \mathbf{p} .
$$

The chemical potentials in the exhibitor indicators under integral in (53) and in the normal multiplier will be reduced, and as a result of simple calculation we will come to such an answer

$$
S=N\left(\frac{3}{2}-\frac{\mu}{T}\right)
$$

Remembering now the definition (50), we get the following differential equation to determine of chemical potential $\mu$

$$
\left(\frac{\partial \mu}{\partial T}\right)_{P}=\frac{\mu}{T}-\frac{3}{2}
$$

Simple integration leads us to the next result

$$
\mu(P, T)=C(P) T-\frac{3}{2} T \ln T,
$$

where the dependence $C(P)$ we can easy find due to the second ratio in expr. (50), i.e.

$$
V=N\left(\frac{\partial \mu}{\partial P}\right)_{T}=N T \frac{d C}{d P} .
$$

Since the Clapeyron-Mendeleev equation $P V=N T$ is in place for the ideal gas, we immediately get that

$$
C(P)=A+\ln P
$$

where $A$ is an constant of integration. Assuming that $A=1$ and substituting expr. (58) to the (56), find the dependency we're going to find

$$
\mu(P, T)=T+T \ln \left(\frac{P}{P_{0}}\right)-\frac{3}{2} T \ln \left(\frac{T}{T_{0}}\right),
$$

where $T_{0}, P_{0}$ are the temperature and the pressure at normal condition, i.e. $T_{0}=300 \mathrm{~K}$, $P_{0}=1 \mathrm{~atm}=10^{5} \mathrm{~Pa}$. That is, for the gas phase, the chemical potential is determined by (59) as

$$
\mu_{2}=T+T \ln \left(\frac{P_{2}}{P_{0}}\right)-\frac{3}{2} T \ln \left(\frac{T}{T_{0}}\right) .
$$


As for the drop of water, it is very problematic to use the gas approximation for it, and in this case it is necessary to apply the equation of the state of Van-der-Waals. As a result, the chemical potential can also be calculated analytically, but now we will not stop there, and move on to the assessment of the time of evaporation, considering for simplicity that $\mu_{1} \sim \mu_{2}$. Note, by the way, that this ratio is quite correct. To estimate the evaporation time according to the general expression (43), we will select the following values of the parameters included in it

$$
\begin{aligned}
& \alpha=70 \frac{\mathrm{erg}}{\mathrm{cm}^{2}}, \quad \mu_{1} \sim \mu_{2}=6 \cdot 10^{-14} \mathrm{erg}, \quad T=300 \mathrm{~K}=4 \cdot 10^{-14} \mathrm{erg}, \\
& R_{0}=5 \cdot 10^{-1} \mathrm{~cm}, \quad \tau_{12}=10^{-10} \mathrm{c}, \delta \approx 10^{-6} \mathrm{~cm} .
\end{aligned}
$$

In the result

$$
\begin{aligned}
& t_{\text {evap }}=\tau_{12} \frac{4 \pi \alpha R_{0}^{2} \delta^{2}}{\left(\frac{\mu_{1}}{4 T}\left(1-\frac{\mu_{2}}{\mu_{1}}\right)^{2}+\frac{\xi \mu_{1}^{2}}{T^{2}}-1\right)\left(\frac{\mu_{1}+\bar{\varepsilon}_{1}}{T}-1\right) \mu_{1} l_{12}^{2}} \approx \\
& \approx 10^{-10} \frac{4 \pi \cdot 70 \cdot 25 \cdot 10^{-2} \cdot 10^{-12}}{3 \cdot 6 \cdot 10^{-14} \cdot 10^{-10}}=\frac{2 \cdot 70 \cdot 25}{3} \approx 1.15 \cdot 10^{3} \mathrm{~s}=20 \mathrm{~min} .
\end{aligned}
$$

That is, a drop of water with a diameter of five millimeters evaporates in about twenty minutes. And then there's. Looking at the equation (39), we clearly see an equation such as a thermal conductivity equation with a temperature-conductivity factor $\chi$, or a diffusion-type equation with a diffusion factor $D$, which is determined by the ratio of the right side of the equation (39), i.e.

$$
D \sim \chi \sim \frac{l_{12}^{2}}{\tau_{12}}=\frac{v_{1 T}^{2} \tau_{12}^{2}}{\tau_{12}}=v_{1 T}^{2} \tau_{12}
$$

This remarkable result is evidence that the evaporation process is purely dissipative and in isotherm conditions is determined by the heterogeneity of chemical potential at the border of contact between liquid and gas. In light of what has been said, it can be argued that according to (62) the described evaporation effect is nothing more than isotherm diffusion. In fact, the assessment (61) of the task of analytical description of the drop evaporation process can be considered solved.

The theoretical approach described above is worth comparing with the approach outlined, for example, in the Fuchs's classic monograph [6]. It is worth noting that this monograph is entirely based on the interpretation of purely empirical dependencies, that is, dependencies obtained experimentally. However, the formulas in it allow us to draw some parallel with the theoretical analysis given a little above. If we enter the Sherwood number according to the formula (see [6,7])

$$
S h=\frac{I_{f}}{2 \pi R D\left(c_{0}-c_{\infty}\right)},
$$

where $I_{f}$ is a speed of evaporation, having the dimension $\frac{g}{s}, D$ is the diffusion coefficient with the dimension $\frac{s m^{2}}{s}, c_{0}$ is the concentration of steam in close proximity to the drop (its dimension is $\left.\frac{\mathrm{g}}{\mathrm{cm}^{3}}\right), c_{\infty}$ is concentration of steam on infinity with the same dimension, in the case of a stationary drop, the Sherwood's number is exactly 2. Using empirical dependence (63) we will find the dependence of the radius of the evaporating drop from time to time. Assuming that 
$I_{f}=\dot{m}$, where the mass of the drop is $m=\rho_{k} V=\frac{4 \pi}{3} \rho_{k} R^{3}$, and accounting that $S h=2$, we find from expr. (63),

$$
4 \pi \rho_{k} R^{2} \dot{R}=4 \pi R D\left(c_{0}-c_{\infty}\right) .
$$

Or

$$
R \dot{R}=\frac{D\left(c_{0}-c_{\infty}\right)}{\rho_{k}} .
$$

Where does the solution come from immediately

$$
R(t)=\sqrt{R_{0}^{2}-D_{e f f} t}
$$

where the effective diffusion coefficient is

$$
D_{e f f}=\frac{D\left(c_{0}-c_{\infty}\right)}{\rho_{k}}
$$

Comparing (65) with our decision (44) we see their full identity. According to the formula (44), the rate of evaporation behaves like

$$
v_{\text {vap }}=|\dot{R}(t)|=\frac{D_{T}}{2 \sqrt{R_{0}^{2}-D_{T} t}} .
$$

It's the right place to go, that when the drop size is reduced, its evaporation rate increases dramatically, which is experimental observe (see an example papers $[8,9]$ ). Dependencies (44) and (67) are illustrated by drawings in Figs. 1, 2.

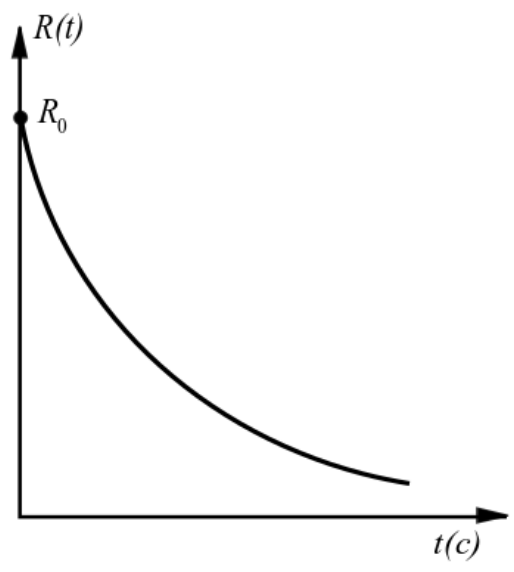

Fig. 1. Schematic representation of the time as dependence of the radius drop

However, our diffusion coefficient (45) and the empirical formula (63) according to (66) are quite different from each other qualitatively. Although in order of magnitude they both give the correct value of the time of evaporation of the stationary drop at condition that in the formulae (66) difference $c_{0}-c_{\infty}$ choose equal $1 \frac{\mathrm{g}}{\mathrm{cm}^{3}}$, and the diffusion coefficient put equal as in our theory value $D=5 \cdot 10^{-5} \frac{\mathrm{cm}^{2}}{\mathrm{~s}}$. This is, in principle, understandable, since the rigorous analytical solution to the problem, based on the equation of preserving the amount of dissipative function (1) and the experimentally obtained dependence (63), is based on different physical assumptions on which the authors rely. 


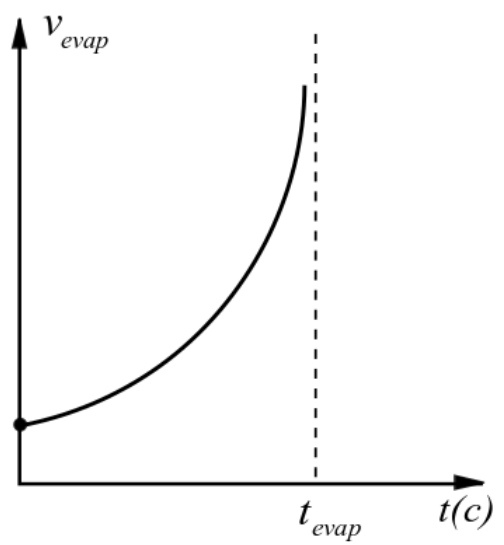

Fig. 2. The time as dependence of the evaporation rate drop

\section{Conclusion}

In the conclusion, it is worth noting three important points.

1. The theory of evaporation of droplets of fine-dispersed environment, based on the condition of preservation of dissipative function, has been built (dissipated energy cannot disappear without a trace, but passes into something).

2. Suggested description of the dynamics of the drop in a high-temperature environment, taking into account its evaporation.

3. The numerical estimates of the optimal size of the drops and their initial speed in the jet are.

\section{References}

[1] E.M.Lifshitz, L.P.Pitaevsky, Physical Kinetics, Vol. 10, Moscow, Nauka, 1989.

[2] L.D.Landau, E.M.Lifshitz, Statistical Physics, Vol. 5, Moscow, Nauka, 1988.

[3] S.O.Gladkov, S.B.Bogdanova, Russian Physics Journal, 61(2018), 833-842. DOI: $10.1007 / \mathrm{s} 11182-018-1467-6$

[4] N.N.Bogolyubov, Selected works on statistical physics, Moscow, Nauka, 1979 (in Russian).

[5] S.O.Gladkov, A collection of tasks on theoretical and mathematical physics, Moscow, Fizmatlit, 2010 (in Russian).

[6] N.A.Fuchs, Evaporation and droplet growth in a gaseous medium, Moscow, Publ. USSR Academy of Sciences, 1958 (in Russian).

[7] H.Green, W.Lane, Particulate clouds: dusts, smokes and mists, Published by E. \& F. N. Spon, London 1957.

[8] V.I.Terekhov, V.V.Terekhov, N.E.Shishkin, K.C.Be, Engineering is a physical journal, 83(2010), no. 5, 829-836. DOI: 10.1007/s10891-010-0410-7 
[9] A.A.Semenov, D.V.Feoktistov, D.V.Zaitsev, G.V.Kuznetsov, O.A.Kabov, Thermophysics and Aeromechanics, 22(2015), 771-774. DOI: 10.1134/S086986431506013X

\title{
К вопросу аналитической оценки времени испарения капли, проходящей сквозь горячую среду
}

Сергей О. Гладков Московский авиационный институт

Москва, Российская Федерация

\begin{abstract}
Аннотация. С помощью кинетического подхода предложено модельное описание процесса испарения капли, движущейся в раскаленной среде. Основное уравнение теории получено благодаря использованию закона сохранения диссипативных функций системы пар - жидкость. Найден диапазон размеров капли, при которых она устойчива. Дано сравнение полученных результатов с известными классическими. Приведены численные оценки размеров мелкодисперсной фазы, при которых имеет место обычное испарение (то есть выполняется условие на число Кнудсена $K n=\frac{l}{R} \ll 1$, где $l$ - длина свободного пробега молекулы, а $R$ - радиус капли).
\end{abstract}

Ключевые слова: диссипативная функция, испарение, длина свободного пробега. 